Discrete Comput Geom 27:195-214 (2002)

DOI: $10.1007 / \mathrm{s} 00454-001-0061-8$

\title{
On the Topology of the Combinatorial Flag Varieties*
}

\author{
A. V. Borovik, ${ }^{1}$ I. M. Gelfand, ${ }^{2}$ and D. A. Stone ${ }^{3}$ \\ ${ }^{1}$ Department of Mathematics, UMIST, \\ PO Box 88, Manchester M60 1QD, England \\ borovik@umist.ac.uk \\ ${ }^{2}$ Department of Mathematics, Rutgers University, \\ New Brunswick, NJ 08903, USA \\ igelfand@math.rutgers.edu \\ ${ }^{3}$ Department of Mathematics, Brooklyn College, \\ 2900 Bedford Avenue, Brooklyn, NY 11210-2889, USA \\ dstone@idt.net
}

\begin{abstract}
We prove that the simplicial complex $\Omega_{n}$ of chains of matroids (with respect to the ordering by the quotient relation) on $n$ elements is shellable. This follows from a more general result on shellability of the simplicial complex of $W$-matroids for an arbitrary finite Coxeter group $W$, and generalises the well-known results by Solomon-Tits and Björner on spherical buildings.
\end{abstract}

\section{Definitions and Main Results}

For any positive integer $n$, let $[n]$ denote the set $\{1, \ldots, n\}$, and let $\mathcal{M}_{n}$ be the set of all matroids on $[n]$ of rank distinct from 0 and $n$. Define a partial ordering on $\mathcal{M}_{n}$ by $M^{\prime} \leq M$ if $M^{\prime}$ is a quotient of $M$. Let $\Omega_{n}$ be the simplicial complex of chains in $\mathcal{M}_{n}$; every simplex $\sigma \in \Omega_{n}$ can be written as

$$
\sigma=\left\langle M_{1}, \ldots, M_{r}\right\rangle,
$$

with all $M_{i} \in \mathcal{M}_{n}$ and

$$
M_{1} \lesseqgtr \cdots \lesseqgtr M_{r} .
$$

Following [9] and [10], we call $\Omega_{n}$ the $n$th combinatorial flag variety.

* The work of the third author was partially supported by PSC-CUNY Research Award 6-69437 and EPSRC Research Grant GR/M24707. 
In contexts where the value of $n$ is not important we omit it from the notation and write $\mathcal{M}$ and $\Omega$.

The works [9] and [10] contain detailed discussions of many remarkable properties of the combinatorial flag variety $\Omega_{n}$. It behaves like a universal, characteristic-free finite geometry. In particular, every matroid on $n$ points is representable in $\Omega_{n}$ in the sense specified in [9] and [10], which is the direct reformulation in combinatorial terms of the classical concept of representability of matroids by means of vector configurations. The present work concentrates on topological and cohomological properties of the combinatorial flag varieties.

It is easy to prove that $\Omega_{n}$ is a purely ( $\left.n-2\right)$-dimensional simplicial complex, that is, it has dimension $n-2$ and every simplex is a face of an $(n-2)$-dimensional simplex (Lemma 2.11 below). [28]:

The main purpose of this paper is to prove the following result, conjectured by White

Theorem 1.1. For $n \geq 3$, the simplicial complex $\Omega_{n}$ is shellable.

Notice that the structure of $\Omega_{2}$ is trivial: it is zero-dimensional and consists of three points.

Theorem 1.1 is a special case of Theorem 1.2 below, which concerns combinatorial flag varieties for arbitrary finite Coxeter groups. As explained in [10], $\Omega_{n}$ can be viewed as the combinatorial flag variety $\Omega_{A_{n-1}}$ for the Coxeter group $\mathrm{A}_{n-1}=\operatorname{Sym}_{n}$. Theorem 1.2 is stated and proven in the framework of the theory of Coxeter matroids, see Section 2. Its statement, however, uses only the most basic concepts of the theory and can be given here in full. We refer the reader to the next section for a more detailed discussion of the concepts involved.

Let $W$ be a finite Coxeter group with the system of standard generators $R$. According to one of its (cryptomorphic) definitions, Coxeter matroid on $W$ (or complete flag matroid on $W$ or $W$-matroid) is a map

$$
\mu: W \longrightarrow W
$$

which satisfies the inequality

$$
w^{-1} \mu(u) \leq w^{-1} \mu(w)
$$

for all $u, w \in W$; $\leq$ here denotes the (strong) Bruhat order on $W$.

The set $\Omega_{W}^{\#}$ of all complete flag matroids on $W$ can be turned into a chamber system in the sense of [26], if we define, for every $r \in R$, the $r$-adjacency $\sim_{r}$ on $\Omega_{W}^{\#}$ by the following rule:

$$
\mu \sim_{r} v
$$

if and only if, for all $w \in W$,

$$
\mu(w)=v(w) \text { or } v(w) r .
$$

We denote by $\Omega_{W}$ the simplicial complex associated with the chamber system $\Omega_{W}^{\#}$, and call it the combinatorial flag variety for $W$. 
Theorem 1.2. Let $W$ be a finite Coxeter group of rank $n \geq 2$. Then the simplicial complex $\Omega_{W}$ is purely $(n-1)$-dimensional, and is shellable.

We do not know whether $\Omega_{W}$ is lexicographically shellable in the sense of Björner [3] or the generalisations thereof such as those in [5] or [23].

The following fact is immediate from Theorem 1.3 of [3].

Corollary 1.3. For $n \geq 2$, the simplicial complex $\Omega_{W}$ is homotopy equivalent to the one-point union of a finite number of $(n-1)$-dimensional spheres.

According to a theorem of Solomon and Tits [25] (see also p. 93 of [15]), any spherical building of rank $n$ is homotopy equivalent to a one-point union of finitely many $(n-1)$ spheres. Also, by Björner [3] spherical buildings are shellable. Our main theorems are thus analogues of these results.

Finally, we discuss in this paper some constructions inspired by, and related to, Kontsevich's graph cohomology [21], [22] (Section 5) and use it to compute the homology of the chain complex of Coxeter matroids defined in [6] (Theorem 6.1).

\section{Coxeter Matroids}

Most results in this section can be found in [6], [8] and [9].

\subsection{Parabolic Subgroups}

Let $W$ be a finite Coxeter group with the set of standard involutive generators $R=$ $\left\{r_{1}, \ldots, r_{n}\right\}$. We denote

$$
P^{i_{1} \cdots i_{k}}=\left\langle\left\{r_{1}, \ldots, r_{n}\right\} \backslash\left\{r_{i_{1}}, \ldots, r_{i_{k}}\right\}\right\rangle ;
$$

the subgroup $P^{i_{1} \cdots i_{k}}$ is known as the (standard) parabolic subgroup of type $i_{1} \cdots i_{k}$.

If $P$ is a parabolic subgroup, we denote by $W^{P}$ the coset space $W / P$.

\subsection{Bruhat Order}

The (strong) Bruhat order $\leq$ on $W$ is defined by the following rule:

Let $u, v \in W$. Then $u \leq v$ if and only if $u$ can be obtained from a reduced expression (that is, a shortest possible expression in terms of the standard generators) $v=$ $r_{i_{1}} \cdots r_{i_{l}}$ for $v$ by deleting some of the generators $r_{i_{j}}$.

For every $w \in W$, we define the $w$-shifted Bruhat order $\leq{ }^{w}$ on $W$ by the rule

$$
u \leq{ }^{w} v \quad \text { if and only if } \quad w^{-1} u \leq w^{-1} v .
$$

We can define a Bruhat order on $W^{P}$ by means of the following fact. 
Lemma 2.1 ([17, Lemma 2.1]; see also Chapter 4 of [12]). Given any $w \in W$ and $a$ coset $x P$ of a parabolic subgroup $P$,

(1) There is a unique element in the coset $x P$ maximal in $x P$ with respect to $\leq^{w}$; we denote it $\max _{w}(x P)$. The coset $x P$ also contains a unique $\leq{ }^{w}$-minimal element, denoted $\min _{w}(x P)$.

(2) Let $x P$ and $y P$ be cosets of $P$. Then the following three conditions are equivalent:

(a) $x^{\prime} \leq{ }^{w} y^{\prime}$ for some $x^{\prime} \in x P$ and $y^{\prime} \in y P$;

(b) $\max _{w}(x P) \leq{ }^{w} \max _{w}(y P)$;

(c) $\min _{w}(x P) \leq{ }^{w} \min _{w}(y P)$.

Now if $x P$ and $y P$ are cosets in $W^{P}$ and $w \in W$ we set

$x P \leq{ }^{w} y P \quad$ if and only if $\max _{w}(x P) \leq{ }^{w} \max _{w}(y P)$.

Lemma 2.2 [16]. Let $P$ and $P_{i}, i=1, \ldots, k$, be parabolic subgroups in $W$ such that $P=\bigcap_{i=1}^{k} P_{i}$. Then, for every $u, v \in W$,

$$
u P \leq v P \quad \text { if and only if } \quad u P_{i} \leq v P_{i} \quad \text { for all } i=1, \ldots, k .
$$

Proof. The proof of a result by Deodhar [16, Lemma 3.6] can be easily adapted to show that $\min (u P) \leq \min (v P)$ if and only if $\min \left(u P_{i}\right) \leq \min \left(v P_{i}\right)$ for all $i=1, \ldots, k$; here $\min (u P)$ denotes the $\leq-$ minimal element of $u P$, etc. Now the result follows from Lemma 2.1.

\subsection{Coxeter Matroids}

If $W$ is a finite Coxeter group and $P$ is a parabolic subgroup in $W$, a subset $M \subseteq$ $W^{P}$ is called a Coxeter matroid (for $W$ and $P$ ) if, for every $w \in W$, it contains a unique element maximal in $M$ with respect to the ordering $\leq^{w}$. If we denote this $\leq^{w}$ maximal element by $\mu(w)$, we come to the equivalent (cryptomorphic) definition: a map

$$
\mu: W \longrightarrow W^{P}
$$

is called a Coxeter matroid (for $W$ and $P$ ) or a matroid map if it satisfies the matroid inequality

$$
\mu(u) \leq^{v} \mu(v) \quad \text { for all } \quad u, v \in W .
$$

Indeed, if $\mu$ satisfies the matroid inequality, then its image $\mu(W)$ is a Coxeter matroid in the sense of the original definition, and vice versa. However, it will be more convenient for us to work with maps. Abusing terminology, we omit the word "Coxeter" from the expression "Coxeter matroid". As will be explained later, matroids in the classical understanding of this term form a special class of Coxeter matroids for the Coxeter group $\mathrm{A}_{n-1}=\mathrm{Sym}_{n}$; we refer to them as ordinary matroids. 
Sometimes we call matroids for a non-maximal parabolic subgroup flag matroids; we reserve the term complete flag matroid for matroids $W \longrightarrow W$ for $W$ and the trivial parabolic subgroup 1.

\subsection{Underlying Flag Matroid}

The first and probably most important operation assigns to every matroid $\mu$ for $W$ and $P$ a matroid for $W$ and 1 (or $W$-matroid).

Lemma 2.3 [13, Lemma 5.15]. A map $\mu: W \longrightarrow W^{P}$ is a matroid if and only if the map $\mu^{*}: W \longrightarrow W$ defined by

$$
\mu^{*}: w \mapsto \max _{w} \mu(w)
$$

is a matroid.

If $\mu$ is a matroid map, then the map $\mu^{*}$ is called the underlying complete flag matroid map for $\mu$.

We use the notation $\mu^{*}=\mathrm{u}(\mu)$.

\subsection{Shifts}

This simple construction is a generalisation to arbitrary Coxeter matroids of the concepts of truncation and Higgs lift for ordinary matroids on [ $n$ ], see Chapter 1 of [12] for details.

Lemma 2.4. Let $P$ and $Q$ be parabolic subgroups of the Coxeter group $W$ and let $\mu: W \longrightarrow W^{P}$ be a matroid for $W$ and $P$. Then the map

$$
\begin{aligned}
\mu^{Q}: W & \rightarrow W^{Q} \\
w & \mapsto \max _{w}(\mu(w)) \cdot Q
\end{aligned}
$$

is a matroid for $W$ and $Q$.

We call the matroid $\mu^{Q}$ the shift of $\mu$ to $W^{Q}$. Notice that $\mathrm{u}(\mu)=\mu^{\langle 1\rangle}$.

Proof. Let $u, w \in W$. Since $\mu(u) \leq{ }^{w} \mu(w)$, we have, by definition of the Bruhat order on $W^{P}$,

$$
\max _{w}(\mu(u)) \leq{ }^{w} \max _{w}(\mu(w)) .
$$

However, then

$$
\mu^{Q}(u)=\max _{w}(\mu(u)) \cdot Q \leq^{w} \max _{w}(\mu(w)) \cdot Q=\mu^{Q}(w)
$$

by Lemma 2.1, which proves that $\mu^{Q}$ is a matroid for $W$ and $Q$. 


\subsection{Concordance}

Let $P$ and $Q$ be parabolic subgroups of $W$. Let

$$
\mu: W \longrightarrow W^{P} \quad \text { and } \quad \nu: W \longrightarrow W^{Q}
$$

be matroids. We say that $\mu$ and $v$ are concordant, if, for every $w \in W$, the intersection of the cosets $\mu(w) \cap \nu(w)$ is non-empty.

It is well known that the intersection of two cosets $x P$ and $y Q$ is either empty or has the form $z(P \cap Q)$ for some $z$. Hence two concordant matroids $\mu$ and $v$ define a map $\lambda: W \longrightarrow W^{P \cap Q}$ by the rule

$$
\lambda: w \mapsto \mu(w) \cap v(w) .
$$

Lemma 2.5. If $\mu$ and $v$ are concordant, then $\lambda$ is a matroid map.

Proof. Let $u, v \in W$. Choose $x \in \mu(v) \cap v(v)$ and $y \in \mu(u) \cap v(u)$. Then $x P=\mu(v)$ and $x Q=v(v)$, and correspondingly $y P=\mu(u)$ and $y Q=v(u)$. Hence $x P \leq^{u} y P$ and $x Q \leq^{u} y Q$. Now it follows from Lemma 2.2 that

$$
x(P \cap Q) \leq^{u} y(P \cap Q),
$$

which means

$$
\lambda(v) \leq^{u} \lambda(u) .
$$

If $\mu$ and $v$ are two concordant Coxeter matroids, we denote the flag matroid $w \mapsto$ $\mu(w) \cap \nu(w)$ by $\mu \vee v$.

Lemma 2.6. Let $P, Q$ and $S$ be parabolic subgroups and let $\mu: W \longrightarrow W^{P}$ be a matroid for $W$ and $P$. Then $\mu$ and its shifts $\mu^{Q}$ and $\mu^{S}$ are pairwise concordant.

Proof. By definition, the cosets $\mu(w), \mu^{Q}(w)=\max _{w}(\mu(w)) \cdot Q$ and $\mu^{S}(w)=$ $\max _{w}(\mu(w)) \cdot S$ have in common the element $\max _{w} \mu(w)$. Hence $\mu, \mu^{Q}$ and $\mu^{S}$ are pairwise concordant.

Lemma 2.7. Let $P, Q$ and $S$ be parabolic subgroups in $W$ and let $\mu: W \longrightarrow W^{P}$ be a matroid for $W$ and $P$. Then

$$
\left(\mu \vee \mu^{Q}\right)^{S}=\mu^{S}
$$

Proof. By definition,

$$
\begin{aligned}
\left(\mu \vee \mu^{Q}\right)^{S}(w) & =\max _{w}\left(\mu(w) \cap \max _{w}(\mu(w)) \cdot Q\right) \cdot S \\
& =\max _{w}(\mu(w)) \cdot S \\
& =\mu^{S}(w) .
\end{aligned}
$$




\subsection{Constituents of a Flag Matroid}

Let $\mu: W \longrightarrow W^{P}$ be a matroid for $W$ and a parabolic subgroup $P=P^{i_{1} \cdots i_{k}}$. Then $P=P^{i_{1}} \cap \cdots \cap P^{i_{k}}$. Denote $\mu^{i_{j}}=\mu^{P_{i_{j}}}, j=1, \ldots, k$.

Lemma 2.8. The matroids $\mu^{i_{1}}, \ldots, \mu^{i_{k}}$ are pairwise concordant and

$$
\mu=\mu^{i_{1}} \vee \cdots \vee \mu^{i_{k}} .
$$

Proof. The matroids $\mu^{i_{j}}$ are pairwise concordant by Lemma 2.6; the property $\mu=$ $\mu^{i_{1}} \vee \cdots \vee \mu^{i_{k}}$ immediately follows from the definition of the operation $\vee$.

We call $\mu_{i_{j}}$ the $i_{j}$ th constituent of $\mu$. We also say that $\mu$ is a flag matroid composed of matroids $\mu^{i_{1}}, \ldots, \mu^{i_{k}}$.

Lemma 2.9. Let $\mu: W \longrightarrow W^{P}$ be a matroid for $W$ and a non-maximal parabolic subgroup $P$ of type $i_{1} \cdots i_{k}$. Let $[n] \backslash\left\{i_{1}, \ldots, i_{k}\right\}=\left\{j_{1}, \ldots, j_{l}\right\}$. Then

$$
\mathrm{u}(\mu)=\mu \vee \mu^{P_{j_{1}}} \vee \cdots \vee \mu^{P_{j_{l}}} .
$$

In particular, the constituents of $\mathrm{u}(\mu)$ are those of $\mu$ together with $\mu^{P_{j_{1}}}, \ldots, \mu^{P_{j_{l}}}$.

Proof. Obvious.

\subsection{Combinatorial Flag Variety}

Let $W$ be a Coxeter group of rank $n$. We turn the set $\Omega^{\#}$ of all matroids $\mu: W \longrightarrow W$ into a chamber system (in the sense of Tits [26]) indexed by the set $[n]$ if we define $i$-adjacency $\sim_{i}$ on $\Omega^{\#}$ by the following rule:

$$
\mu \sim_{i} v
$$

if and only if, for all $w \in W$,

$$
\mu(w)=v(w) \text { or } v(w) r_{i} .
$$

We denote by $\Omega_{W}$ the simplicial complex associated with $\Omega_{W}^{\#}$ and call it the combinatorial flag variety for $W$. We usually suppress the subscript $W$ in $\Omega^{\#}=\Omega_{W}^{\#}$.

Lemma 2.10. Let

$$
\mu=\mu^{1} \vee \cdots \vee \mu^{n}
$$

and

$$
v=v^{1} \vee \cdots \vee v^{n}
$$

be decompositions of complete flag matroids $\mu$ and $v$ into constituents. Then $\mu \sim_{i} v$ if and only if $\mu^{j}=v^{j}$ for all $j \neq i$. 
Proof. The generator $r_{i}$ belongs to all maximal parabolic subgroups $P^{j}$ for $j \neq i$, hence if $\mu \sim_{i} v$, then $\mu(w)=v(w)$ or $v(w) r_{i}$ by definition of adjacency and in both cases $\mu(w) P^{j}=v(w) P^{j}$, hence $\mu^{j}=v^{j}$ for all $j \neq i$. On the other hand, if $\mu^{j}=v^{j}$ for all $w \in W$ and $j \neq i$, we have

$$
\mu(w)^{-1} v(w) \in \bigcap_{j \neq i} P^{j}=\left\langle r_{i}\right\rangle,
$$

hence $v(w) \in \mu(w)\left\langle r_{i}\right\rangle$ for all $w \in W$, which means $\mu \sim_{i} \nu$.

Therefore the simplicial complex $\Omega$ associated with the chamber system $\Omega^{\#}$ can be canonically identified with the set of all tuples $\left\langle\mu^{i_{1}}, \ldots, \mu^{i_{k}}\right\rangle$ of pairwise concordant matroids $\mu^{i_{j}}$ for distinct maximal parabolic subgroups $P^{i_{j}}$, ordered by inclusion $\subseteq$. The intersection $\mu \cap v$ of two simplices $\mu=\left\langle\mu^{i_{1}}, \ldots, \mu^{i_{k}}\right\rangle$ and $v=\left\langle v^{i_{1}}, \ldots, v^{i_{l}}\right\rangle$ has the usual set-theoretic meaning.

Lemma 2.11. Let $n$ be the rank of $W$. If $k \geq 2$, then $\Omega_{W}$ is a purely $(n-1)$-dimensional complex.

Proof. It follows from Lemma 2.9 that every matroid $\mu$ is a face of its underlying matroid $\mathrm{u}(\mu)$.

\subsection{Weak Map Ordering}

We define the weak map ordering « on the set of all matroids for $W$ and $P$ by the following rule: $\mu \ll v$ if and only if $\mu(w) \leq w v(w)$ for all $w \in W$. It is easy to see that for finite Coxeter groups $W$ this is equivalent to inclusion of images $\mu[W] \subseteq \nu[W]$.

The name is justified by the following observation: if $M$ and $N$ are two (ordinary) matroids on $[n]$ of the same rank $k$, then $M \ll N$ in the sense of our definition if and only if every basis of $M$ is a basis of $N$, that is, if the identity map on [n] is a weak map from $N$ to $M$.

Let $\mu: W \longrightarrow W^{P}$ be a matroid and let $Q$ be a parabolic subgroup. The following lemma improves on Lemma 2.6 and shows that the shift $\mu^{Q}$ is the "biggest" matroid for $W$ and $Q$ which is concordant with $\mu$.

Lemma 2.12. Let $\mu: W \longrightarrow W^{P}$ and $v: W \longrightarrow W^{Q}$ be concordant matroids. Then

$$
v \ll \mu^{Q} \text {. }
$$

Proof. Let $w \in W$ and $x$ be a common element in $\mu(w) \cap v(w)$. Then $x \leq w$ $\max _{w}(\mu(w))$, hence

$$
v(w)=x Q \leq^{w} \max _{w}(\mu(w)) \cdot Q=\mu^{Q}(w)
$$

by Lemma 2.1. Therefore $v \ll \mu^{Q}$. 


\subsection{Expansion}

Another tool for our study of a shelling for $\Omega$ will be a map

$$
\exp ^{j}: \Omega^{\#} \longrightarrow \Omega^{\#}
$$

defined for every $j \in[n]$ by the following rule: to obtain $\exp ^{j}(\mu)$, we replace the constituent $\mu^{j}$ in $\mu=\left\langle\mu^{1}, \ldots, \mu^{j}, \ldots, \mu^{n}\right\rangle$ by

$$
\left(\mu^{1} \vee \cdots \vee \mu^{j-1} \vee \mu^{j+1} \vee \cdots \vee \mu^{n}\right)^{P_{j}},
$$

the largest matroid for $W$ and $P^{j}$ concordant with the rest of the constituents $\mu^{i}, i \neq j$. We call $\exp ^{j}(\mu)$ the $j$ th expansion of $\mu$.

Notice that $\mu \sim_{j} \exp ^{j}(\mu)$. The expansion $\exp ^{j}(\mu)$ is uniquely determined by the property that $v^{j} \ll\left(\exp ^{j}(\mu)\right)^{j}$ for all $v \sim_{j} \mu$.

Lemma 2.13. Let $\mu: W \longrightarrow W^{P}$ be a matroid for $W$ and a non-maximal parabolic subgroup $P$ of type $i_{1} \cdots i_{k}$. Then, for all $j \notin\left\{i_{1}, \ldots, i_{k}\right\}$,

$$
\exp ^{j}(\mathrm{u}(\mu))=\mathrm{u}(\mu)
$$

Proof. Let $[n] \backslash\left\{i_{1}, \ldots, i_{k}\right\}=\left\{j_{1}, \ldots, j_{l}\right\}$. By Lemma 2.9,

$$
\mathrm{u}(\mu)=\mu \vee \mu^{P_{j_{1}}} \vee \cdots \vee \mu^{P_{j_{l}}}
$$

the result now follows from the definition of $\exp ^{j}$ and Lemma 2.7.

\section{Relation to Ordinary Matroids}

In this section we explain why and how matroids in the classical meaning of this word can be treated as Coxeter matroids. This will explain, in particular, why Theorem 1.1 is a special case of Theorem 1.2.

Detailed proofs and discussion of results in this section can be found in [7] and in Chapters 1 and 4 of [12].

We work with the symmetric group $W=\mathrm{Sym}_{n}$ viewed as the Coxeter group of type $\mathrm{A}_{n-1}$. In particular, the standard involutive generators of $W$ are transpositions $r_{i}=$ $(i, i+1), i=1,2, \ldots, n-1$.

Let $\mathcal{P}_{n, k}$ be the set of all $k$-element subsets in [n]. Since the parabolic subgroup $P^{k}=\left\langle r_{i} \mid i \neq k\right\rangle$ is the setwise stabiliser in $W$ of the set $[k]=\{1,2, \ldots, k\}$, the set $\mathcal{P}_{n, k}$ can be identified with the factor set $W^{P^{k}}=W / P^{k}$.

We introduce a partial ordering $\leq$ on $\mathcal{P}_{n, k}$ (the Gale order) as follows. Let $A, B \in \mathcal{P}_{n, k}$, where

$$
A=\left\{i_{1}, \ldots, i_{k}\right\}, \quad i_{1}<i_{2}<\cdots<i_{k},
$$


and

$$
B=\left\{j_{1}, \ldots, j_{k}\right\}, \quad j_{1}<j_{2}<\cdots<j_{k},
$$

then we set

$$
A \leq B \quad \text { if and only if } \quad i_{1} \leq j_{1}, \ldots, i_{k} \leq j_{k} .
$$

It can be checked (see [12] for details) that this order coincides with the (strong) Bruhat order on $W^{P^{k}}$. We use the notation $\leq^{w}$ with the same meaning as before.

Theorem 3.1 [18]. Let $\mathcal{B} \subseteq \mathcal{P}_{n, k}$. Then $\mathcal{B}$ is the set of bases of a matroid if and only if $\mathcal{B}$ satisfies the following Maximality Property:

for every $w \in \operatorname{Sym}_{n}$ the collection $\mathcal{B}$ contains a unique member $A \in \mathcal{B}$ maximal in $\mathcal{B}$ with respect to $\leq^{w}$, that is, $B \leq{ }^{w}$ A for all $B \in \mathcal{B}$.

We call $A$ the $w$-maximal basis in $\mathcal{B}$.

Interestingly, a definition of matroid in terms of orderings was anticipated by Boruvka [14] before the invention of matroids. It was introduced into mainstream combinatorial theory by Gale [18] as a solution of the problem of optimal assignment in applied combinatorics, and then later but independently rediscovered, in the wider context of Coxeter matroids, by Gelfand and Serganova [19], [20].

\subsection{Matroids as Maps}

Now let $\mathcal{B}$ be the set of bases of a matroid $M$ of $\operatorname{rank} k$ on $[n]$. We can define a map

$$
\mu: \operatorname{Sym}_{n} \longrightarrow \mathcal{P}_{n, k}
$$

assigning to each $w \in \operatorname{Sym}_{n}$ the element $A \in \mathcal{B}$ maximal in $\mathcal{B}$ with respect to $\leq^{w}$. Obviously this map satisfies the inequality

$$
\mu(u) \leq{ }^{w} \mu(w)
$$

for all $u, w \in \operatorname{Sym}_{n}$. Since any $k$-element set $B \in \mathcal{P}_{n, k}$ can be made maximal in $\mathcal{P}_{n, k}$ after some reordering of the symbols $1,2, \ldots, n$, we have $\mu\left[\operatorname{Sym}_{n}\right]=\mathcal{B}$. Vice versa, the image of every map $\mu$ from $\operatorname{Sym}_{n}$ to $\mathcal{P}_{n, k}$ which satisfies the above inequality for all $u$ and $w$ is the set of bases of a matroid.

Hence (ordinary) matroids of rank $k$ on $[n]$ can be interpreted as matroid maps $\mu: W \longrightarrow W^{P^{k}}$ for the Coxeter group $W=\mathrm{A}_{n-1}$ and the parabolic subgroup $P^{k}$.

\subsection{Flags}

A flag $F$ is a strictly increasing sequence

$$
F_{1} \subset F_{2} \subset \cdots \subset F_{m}
$$


of finite sets. Denote by $k_{i}$ the cardinality of the set $F_{i}$; the $m$-tuple $\left(k_{1}, \ldots, k_{m}\right)$ is called the rank of $F$. We write $F=\left(F_{1}, \ldots, F_{m}\right)$; the set $F_{i}$ is called the $i$ th constituent, or the constituent of rank $k_{i}$, of the flag $F$. The set of all flags of rank $\left(k_{1}, \ldots, k_{m}\right)$ in $[n]$ is denoted $\mathcal{F}_{n}^{k_{1} \cdots k_{m}}$.

For every $w \in \operatorname{Sym}_{n}$ we define the Gale order $\leq^{w}$ on $\mathcal{F}_{n}^{k_{1} \cdots k_{m}}$ constituentwise: if $F=\left(F_{1}, \ldots, F_{m}\right)$ and $G=\left(G_{1}, \ldots, G_{m}\right)$ are two flags, we set $F \leq^{w} G$ if and only if $F_{i} \leq{ }^{w} G_{i}$ for all $i=1, \ldots, m$.

\subsection{Flag Matroids}

A set $\mathcal{F}$ of flags of rank $\left(k_{1}, \ldots, k_{m}\right)$ is called a flag matroid if and only if $\mathcal{F}$ satisfies the Maximality Property:

for every $w \in \operatorname{Sym}_{n}$ the set $\mathcal{F}$ contains a unique element maximal in $\mathcal{F}$ with respect to the ordering $\leq{ }^{w}$.

For any set $\mathcal{F}$ of flags, we call the collection of $i$ th constituents $F_{i}$ for $F \in \mathcal{F}$ the $i$ th constituent of $\mathcal{F}$. It follows immediately from this definition of flag matroid that the $i$ th constituent of a flag matroid $\mathcal{F}$ is the set $\mathcal{B}_{i}$ of bases of a matroid $M_{i}$ of rank $k_{i}$, called the $i$ th constituent matroid of $\mathcal{F}$. In particular, if $\left(F_{1}, \ldots, F_{m}\right)$ is the unique maximal element with respect to $\leq^{w}$, then for each $i, 1 \leq i \leq m, F_{i}$ is the unique maximal basis of the constituent matroid $M_{i}$ with respect to $\leq{ }^{w}$.

Notice that every matroid is a flag matroid and that $\mathcal{P}_{n, k}=\mathcal{F}_{n}^{k}$.

\subsection{Matroid Quotients}

Let $M$ and $M^{\prime}$ be matroids on the same set [n]. We say that $M^{\prime}$ is a quotient of $M$ if every circuit of $M$ is a union of circuits of $M^{\prime}$. An equivalent statement found in some texts is that the identity map on $[n]$ is a strong map from $M$ to $M^{\prime}$. There are many equivalent characterisations of quotients or strong maps, see Proposition 7.4.7 and Chapter 8 of [27]. We will need the following facts about quotients. If $M^{\prime}$ is a quotient of $M$ and the two matroids are not equal, then $\operatorname{rank}\left(M^{\prime}\right)<\operatorname{rank}(M)$. Furthermore, every basis

of $M$ contains a basis of $M^{\prime}$, and every basis of $M^{\prime}$ is contained in a basis of $M$ (the last statement is a restatement of the fact that a strong map is also a weak map [24, Lemma 8.1.7]).

\subsection{Flag Matroids Are Coxeter Matroids}

We now come to the main theorem characterising flag matroids.

Theorem 3.2. A set $\mathcal{F}$ of flags of rank $\left(k_{1}, \ldots, k_{m}\right)$ is a flag matroid if and only if

(1) each constituent $M_{i}$ of $\mathcal{F}$ is a matroid,

(2) $M_{i}$ is a quotient of $M_{j}$ for all $i<j$ and 
(3) if the bases $B_{i}$ of $M_{i}, i=1, \ldots, m$, form a flag

$$
B_{1} \subset \cdots \subset B_{m}
$$

then this flag belongs to $\mathcal{F}$.

Corollary 3.3. A collection of matroids of distinct ranks forms a flag matroid if and only if, for every $w \in \mathrm{Sym}_{n}$, the $\leq{ }^{w}$-maximal bases of each matroid form a flag.

A flag matroid is complete if it contains constituents of every possible rank. A complete flag of sets

$$
\left[i_{1}\right] \subset\left[i_{1}, i_{2}\right] \subset \cdots \subset\left[i_{1}, \ldots, i_{n}\right]
$$

can be identified with the permutation

$$
\left(\begin{array}{cccc}
1 & 2 & \cdots & n \\
i_{1} & i_{2} & \cdots & i_{n}
\end{array}\right)
$$

The Gale order on $\mathcal{F}_{n}^{1 \cdots n}$ becomes the (strong) Bruhat order on $\mathrm{Sym}_{n}$ viewed as the Coxeter group $\mathrm{A}_{n-1}$. Given a complete flag matroid $M=\left\langle M^{1}, \ldots, M^{n}\right\rangle$, we can introduce the map $\mu: \operatorname{Sym}_{n} \longrightarrow \operatorname{Sym}_{n}$ by setting

$$
\mu: w \mapsto w \text {-maximal flag in } M,
$$

which, as shown in [9], is a matroid map with respect to the Bruhat ordering. Vice versa, every matroid map $\mu: \operatorname{Sym}_{n} \longrightarrow \mathrm{Sym}_{n}$ is a complete flag matroid.

It is easy to see that two matroid maps $\mu, \mu^{\prime}: \operatorname{Sym}_{n} \longrightarrow \operatorname{Sym}_{n}$ are $j$-adjacent if and only if the $i$ th constituents of the corresponding complete flag matroids $\mathcal{F}$ and $\mathcal{F}^{\prime}$ coincide for all $i \neq j$.

This identifies the combinatorial flag variety $\Omega_{n}$ with $\Omega_{\mathrm{A}_{n-1}}$. Hence Theorem 1.1 is a special case of Theorem 1.2.

\section{Proof of Theorem 1.2}

Let $\Omega=\Omega_{W}$. We need to prove that $\Omega$ is shellable. Our proof uses ideas of Björner [2]. However, we have not been able to prove that $\Omega$ is lexicographically shellable, either in the sense of [2] or its generalisations.

We have already proved (Lemma 2.11$)$ that $\Omega$ is purely $(n-1)$-dimensional, where $n$ is the rank of $W$.

Define a partial ordering $\prec$ on the set $\Omega^{\#}$ of $(n-1)$-simplices of $\Omega$ in the following way. Let

$$
\mu=\left\langle\mu^{1}, \ldots, \mu^{n}\right\rangle
$$

and

$$
v=\left\langle v^{1}, \ldots, v^{n}\right\rangle
$$


be in $\Omega^{\#}$; then $\mu \prec v$ if there exists $j \in[n]$ such that

- $\mu^{i}=v^{i}$ for $i=0, \ldots, j-1$;

- $\mu^{j} \neq v^{j}$

- $\mu^{j} \ll v^{j}$ in the weak map ordering.

Choose a linear ordering $\mu_{1}, \ldots, \mu_{t}$ of $\Omega^{\#}$ such that if $\mu_{i} \prec \mu_{j}$, then $i>j$. We shall prove that $\mu_{1}, \ldots, \mu_{t}$ is a shelling of $\Omega$. To do so it will be enough to show (see Björner p. 163 of [2]):

$$
\begin{aligned}
& \text { If } \mu_{p}, \mu_{q} \in \Omega^{\#} \text { with } p<q \text {, then there exists } r \in[q-1] \text { such that } \\
& \qquad \mu_{p} \cap \mu_{q} \subseteq \mu_{r} \cap \mu_{q} \text { and } \mu_{r} \text { and } \mu_{q} \text { are adjacent. }
\end{aligned}
$$

Write $\mu_{p}=\left\langle\mu_{p}^{1}, \ldots, \mu_{p}^{n}\right\rangle$ and similarly for $q$. Let $a$ be the largest integer such that $0 \leq a \leq n$ and $\mu_{p}^{i}=\mu_{q}^{i}$ for $0 \leq i \leq a$.

Define a simplex $\sigma_{r} \in \Omega^{\#}$ by

$$
\mu_{r}=\exp ^{a+1}\left(\mu_{q}\right)
$$

Notice that it is not the case that $\mu_{p}^{a+1} \ll \mu_{q}^{a+1}$; indeed, otherwise $\mu_{p} \prec \mu_{q}$ and $q<p$, a contradiction. Therefore $\mu_{r} \neq \mu_{q}$ and $\mu_{q} \prec \mu_{r}$ by Lemma 2.12, so $r<q$. Also $\mu_{r}$ and $\mu_{q}$ are adjacent,

$$
\mu_{r} \cap \mu_{q}=\left\langle\mu_{q}^{1}, \ldots, \widehat{\mu_{q}^{a+1}}, \ldots, \mu_{q}^{n-1}\right\rangle ;
$$

and since $\mu_{p}^{a+1} \neq \mu_{q}^{a+1}$,

$$
\mu_{r} \cap \mu_{q} \supseteq \mu_{p} \cap \mu_{q}
$$

We have thus verified that the ordering $\mu_{1}, \ldots, \mu_{t}$ satisfies the definition a shelling of $\Omega$.

It now follows that $\Omega$ has the homotopy type of the one-point union of a certain number $h$ of $(n-1)$-dimensional spheres [3, Theorem 1.3]. Moreover, let

$$
\Omega^{b}=\left\{\mu \in \Omega^{\#} \mid \text { for all } i=1, \ldots, n, \mu \neq \exp ^{i}(\mu)\right\} ;
$$

then it follows from Theorem 7.7.2 of [4] that $h=\left|\Omega^{b}\right|$.

Notice that, for every $w \in W$, the constant map

$$
W \longrightarrow\{w\}
$$

is a matroid map. The set $\mathbf{A}$ of all such maps forms a subcomplex in $\Omega$ isomorphic to the Coxeter complex for $W$. Obviously, $\mathbf{A} \subseteq \Omega^{b}$. In particular, this yields a (very crude) lower bound for the rank of $H^{n-1}(\Omega, \mathbb{Z})$.

Theorem 4.1. The rank of $H^{n-1}(\Omega, \mathbb{Z})$ is at least $|W|$.

We mention here, without proof, a much better lower bound which can be obtained in the case of ordinary matroids (that is, in the case $W=\mathrm{Sym}_{n}$ ). 
Theorem 4.2. In case $W=\operatorname{Sym}_{n}$ and $n \geq 3$, the rank of $H^{n-2}\left(\Omega_{n}, \mathbb{Z}\right)$ is at least

$$
\frac{(n !)^{2}}{2^{n-1} \cdot n}+n !
$$

The proof will appear elsewhere; it involves a direct construction of a family of flag matroids in $\Omega_{n}^{b}$.

Notice also, that, by analogy with the simplicial complex $\Omega_{n}$, one can construct the complex $\Omega_{n}^{\text {rep }}$ consisting of complete flag matroids representable in a vector space in the sense of [11]. It can be shown that $\Omega_{n}^{\text {rep }}$ is shellable; the proof will appear elsewhere.

The following generalisation of Theorem 1.2 will be useful in some future applications. For any subcomplex $\Delta$ of $\Omega$, set $\Delta^{\#}=\Delta \cap \Omega^{\#}$.

Proposition 4.3. Let $\Delta$ be a subcomplex of $\Omega$ such that if $\mu<\Delta$, then $\mathrm{u}(\mu)<\Delta$. Then $\Delta$ is shellable. Moreover, $H_{n-1}(\Delta, \mathbb{Z})$ has rank $\left|\Delta^{\#} \cap \Omega^{\mathrm{b}}\right|$. In particular, if $\Delta^{\#} \cap \Omega^{\mathrm{b}}=\emptyset$, then $\Delta$ is contractible.

Proof. Since $\mathrm{u}(\mu)<\Delta$ for $\mu<\Delta$, it easily follows that $\Delta$ is purely $(n-1)$ dimensional. Let

$$
\mu=\left\langle\mu^{1}, \ldots, \mu^{n}\right\rangle \in \Delta^{\#}
$$

and $j \in[n]$. Set

$$
\partial_{j}(\mu)=\left\langle\mu^{1}, \ldots, \widehat{\mu^{j}}, \ldots, \mu^{n}\right\rangle
$$

(that is, $\mu^{j}$ is deleted from $\mu$ ). Then $\partial_{j}(\mu) \in \Delta$, so also $\mathrm{u}\left(\partial_{j} \mu\right) \in \Delta$. Now

$$
\mathrm{u}\left(\partial_{j} \mu\right)=\exp ^{j}(\mu)
$$

The proof of Theorem 1.2 can now be used to prove the proposition.

\section{Comments on Graph Cohomology}

One of our reasons for undertaking this study was to try to define in the context of matroids something akin to Kontsevich's graph cohomology, $\Gamma H^{*}$ ([21], [22]; see also [1]). Roughly speaking, $\Gamma H^{*}$ is the cohomology of a chain complex $\left(\Gamma C^{*}, d_{\Gamma}\right)$; where $\Gamma C^{*}$ has in degree $n$ one generator for each graph which has $n$ edges, and the coboundary operator $d_{\Gamma}$ assigns to every graph $\Gamma$ the sum of all graphs $\Gamma^{\prime}$ which can be reduced to $\Gamma$ by contracting one edge.

The fact that contracting an edge of a graph corresponds to a strong map of the corresponding matroids suggests that $\Omega_{n}=\Omega_{\mathrm{A}_{n-1}}$ might be a convenient context in which to look for a generalisation of graph cohomology. However, the standard simplicial coboundary operator $d$ in $\Omega_{n}$ would assign to a matroid $M$ a cochain of the form

$$
d(M)=\sum_{\substack{M<M^{\prime} \\ \operatorname{rank}\left(M^{\prime}\right)=\operatorname{rank}(M)+1}} \pm\left\langle M, M^{\prime}\right\rangle+\sum_{\substack{M^{\prime}<M \\ \operatorname{rank}\left(M^{\prime}\right)=\operatorname{rank}(M)-1}} \pm\left\langle M^{\prime}, M\right\rangle,
$$


whereas Kontsevich's formula for $d_{\Gamma}$ more closely resembles a coboundary operator of the form

$$
\delta(M)=\sum \pm\left\langle M, M^{\prime}\right\rangle
$$

where the sum is taken over certain matroids $M^{\prime}$ such that $M<M^{\prime}$ and $\operatorname{rank}\left(M^{\prime}\right)=$ $\operatorname{rank}(M)+1$.

This observation led us to investigate the following configuration.

Let $\Omega=\Omega_{W}$ and $\Sigma=\Omega \backslash \Omega^{b}$. Consider the cochain complex

$$
C^{*}(\Sigma)=C^{*}(\Sigma, \mathbb{Z})
$$

with the coboundary operator $\delta$ given by

$$
\delta(\mu)=d_{\Omega[u(\mu)]}(\mu),
$$

where $\mathrm{u}(\mu)$ is the underlying complete flag matroid of $\mu$, and $d_{\Omega[\mathrm{u}(\mu)]}$ is the usual simplicial coboundary operator in the complex $\Omega[u(\mu)]$ consisting of $u(\mu)$ and its faces.

Even though $\Sigma$ is contractible, and so has the cohomology groups of a point with respect to the standard coboundary operator, it does not follow that its cohomology groups with respect to a non-standard coboundary operator are also the same as those of a point. For example, with the coboundary operator which is 0 in every degree, the cohomology groups of $\Sigma$ are the same as its cochain groups.

We shall prove:

Theorem 5.1. The cochain complex $\left(C^{*}(\Sigma), \delta\right)$ has the cohomology groups of a point:

$$
H^{*}\left(C^{*}(\Sigma), \delta\right)= \begin{cases}Z & \text { in degree } 0 \\ 0 & \text { in all other degrees. }\end{cases}
$$

Proof. Throughout this proof, let an arbitrary orientation of every simplex $\sigma$ of $\Sigma$ be fixed; that is, an ordering of its vertices up to even permutations. We use the same symbol $\sigma$ to denote an oriented and unoriented simplex.

We write $\sigma^{\prime} \leq \sigma$ and $\sigma^{\prime}<\sigma$ if $\sigma^{\prime}$ is a face (respectively, proper face) of the simplex $\sigma$.

To every ordered pair $\left(\sigma, \sigma^{\prime}\right)$ of oriented simplices of $\Sigma$ is assigned an incidence number $\varepsilon\left(\sigma, \sigma^{\prime}\right) \in\{0,1,-1\}$ by the rules:

(1) $\varepsilon\left(\sigma, \sigma^{\prime}\right)=0$ if $\sigma$ is not a face of $\sigma^{\prime}$ or if $\operatorname{dim}(\sigma) \neq \operatorname{dim}\left(\sigma^{\prime}\right)-1$.

(2) If $\sigma$ and $\sigma^{\prime}$ have vertex sets $\left\langle\mu^{i_{0}}, \ldots, \mu^{i_{r}}\right\rangle$ and $\left\langle\mu^{i_{j}}, \mu^{i_{0}}, \ldots, \mu^{i_{r}}\right\rangle$, respectively, ordered so that $\left\langle\mu^{i_{0}}, \ldots, \mu^{i_{r}}\right\rangle$ gives the chosen orientation of $\sigma$, then $\varepsilon\left(\sigma, \sigma^{\prime}\right)$ equals 1 or -1 according as $\left\langle\mu^{i_{j}}, \mu^{i_{0}}, \ldots, \mu^{i_{r}}\right\rangle$ does or does not agree with the chosen orientation of $\sigma^{\prime}$.

Let

$$
C^{*}(\Sigma)=C^{*}(\Sigma ; \mathbb{Z})
$$

be the graded free abelian group of integer-valued cochains on $\Sigma$. We identify an oriented simplex $\sigma$ with the cochain defined by its values on oriented simplices according to the 
rule $\sigma\left(\sigma^{\prime}\right)$ equals 1 if $\sigma=\sigma^{\prime}$ and 0 otherwise. Thus $\Sigma$ is identified with a basis of $C^{*}(\Sigma)$. The standard coboundary operator

$$
d: C^{*}(\Sigma) \longrightarrow C^{*}(\Sigma)
$$

is the linear transformation determined by the rule

$$
d \sigma\left(\sigma^{\prime}\right)=\varepsilon\left(\sigma, \sigma^{\prime}\right) .
$$

As is well known, $d \circ d=0$; in other words, $\left(C^{*}(\Sigma), d\right)$ is a cochain complex.

In this proof we are concerned with a different coboundary operator $\delta$ on $C^{*}(\Sigma)$, defined by $\delta(\sigma)=d_{\Omega[\mathrm{u}(\sigma)]}(\sigma)$; explicitly,

$$
\delta \sigma\left(\sigma^{\prime}\right)= \begin{cases}\varepsilon\left(\sigma, \sigma^{\prime}\right) & \text { if } \quad \sigma^{\prime} \leq \mathrm{u}(\sigma), \\ 0 & \text { otherwise. }\end{cases}
$$

We restate Theorem 5.1 in the following form. Note that once we have shown that $\delta \circ \delta=0$, it will follow that the cohomology

$$
H^{*}\left(C^{*}(\Sigma), \delta\right)=\operatorname{ker}(\delta) / \operatorname{Im}(\delta)
$$

is a well-defined graded abelian group.

\section{Theorem 5.2.}

(1) $\left(C^{*}(\Sigma), \delta\right)$ is a cochain complex; that is, $\delta \circ \delta=0$.

(2) $H^{*}\left(C^{*}(\Sigma), \delta\right)$ is equal to the cohomology of a point:

$$
H^{*}\left(C^{*}(\Sigma), \delta\right)= \begin{cases}Z & \text { in degree } 0 \\ 0 & \text { in all other degrees }\end{cases}
$$

Proof. The proof includes Lemmas 5.3-5.6 and Corollary 5.7.

Lemma 5.3. If $\sigma \in \Sigma$, then $\mathrm{u}(\sigma) \in \Sigma$.

Proof. Follows from Lemma 2.13.

Let $\sigma_{1}, \ldots, \sigma_{q}$ be an ordering of $\Sigma^{\#}$ such that if $\sigma_{i} \prec \sigma_{j}$, then $i>j$. Recall that for any $\sigma \in \Omega, \Omega[\sigma]$ is the subcomplex of $\Omega$ consisting of $\sigma$ and its faces. For $i=1, \ldots, q$ set

$$
\begin{aligned}
\Sigma(i) & =\bigcup_{j=1}^{i} \Omega\left[\sigma_{j}\right], \\
S_{i} & =\Sigma(i) \backslash \Sigma(i-1) .
\end{aligned}
$$

Observe that the $S_{i}$ are pairwise disjoint, and

$$
\Sigma=\bigcup_{i=1}^{q} S_{i}
$$




\section{Lemma 5.4.}

(1) $\Sigma(1)$ is contractible.

(2) $\Sigma(i)$ has $\Sigma(i-1)$ as deformation retract.

Proof. (1) is obvious, (2) follows from [3], since $\Sigma(i) \cap \Omega^{b}=\emptyset$.

Lemma 5.5. Let $\sigma \in S_{i}$; then $\mathrm{u}(\sigma)=\sigma_{i}$.

Proof. If $\sigma=\sigma_{i}$ is of maximal dimension, then $\mathrm{u}(\sigma)=\sigma_{i}$. Therefore we can assume that $\sigma<\sigma_{i}$ is a proper face of $\sigma_{i}$. Suppose that $\mathrm{u}(\sigma) \neq \sigma_{i}$. Then it follows from the description of constituents in $\mathrm{u}(\sigma)$ (Lemma 2.13) and the maximality of shifts with respect to the weak map ordering (Lemma 2.12) that $\sigma_{i} \prec \mathrm{u}(\sigma)$ by definition of $\prec$; so $\mathrm{u}(\sigma)=\sigma_{j}$ for some $j<i$; but now $\sigma \in \Sigma(j)$, a contradiction.

Let

$$
C^{*}(i)=C^{*}(\Sigma(i), \Sigma(i-1) ; \mathbb{Z})
$$

be the graded free abelian group of cochains on $\Sigma(i)$ which vanish on $\Sigma(i-1)$. Then $S_{i}$ is a basis of $C^{*}(i)$. Let $d_{i}$ be the standard coboundary operator on $C^{*}(i)$ : for $\sigma, \sigma^{\prime} \in S_{i}$,

$$
d_{i} \sigma\left(\sigma^{\prime}\right)= \begin{cases}\varepsilon\left(\sigma, \sigma^{\prime}\right) & \text { if } \sigma^{\prime} \in S_{i}, \\ 0 & \text { otherwise. }\end{cases}
$$

Then $\left(C^{*}(i), d_{i}\right)$ is a cochain complex. Set

$$
\left(C^{*}(\oplus), d_{\oplus}\right)=\left(\bigoplus_{i=1}^{q} C^{*}(i), \sum d_{i}\right),
$$

this is also a cochain complex. Let

$$
\iota: \Sigma \longrightarrow \bigcup_{i=1}^{q} S_{i}
$$

be the identity map. Then $\iota$ induces an isomorphism of graded free abelian groups,

$$
\iota^{*}: C^{*}(\Sigma) \longrightarrow C^{*}(\oplus)
$$

Lemma 5.6. $\quad \iota^{*} \circ \delta=d_{\oplus} \circ \iota^{*}$.

Proof. In view of the formulae for $\delta$ and $d_{\oplus}$ it is enough to prove that for every $\sigma \in \Sigma$, $\delta(\sigma)$ and $d_{\oplus}(\sigma)$ have the same support. Say $\operatorname{dim}(\sigma)=r$ and that $\sigma \in S_{i}$. Then the support

$$
\operatorname{supp}(\delta \sigma)=\left\{\sigma^{\prime} \in \Sigma \mid \sigma<\sigma^{\prime} \leq \mathrm{u}(\sigma) \text { and } \operatorname{dim}\left(\sigma^{\prime}\right)=r+1\right\}
$$


while, since $\operatorname{supp}\left(d_{j} \sigma\right)=\emptyset$ for $j \neq i$,

$$
\begin{aligned}
\operatorname{supp}\left(d_{\oplus} \sigma\right) & =\bigcup_{k=1}^{q} \operatorname{supp}\left(d_{k} \sigma\right) \\
& =\operatorname{supp}\left(d_{i} \sigma\right) \\
& =\left\{\sigma^{\prime \prime} \in S_{i} \mid \sigma<\sigma^{\prime \prime} \text { and } \operatorname{dim}\left(\sigma^{\prime \prime}\right)=r+1\right\}
\end{aligned}
$$

For every $\sigma^{\prime} \in \operatorname{supp}(\delta \sigma), \mathrm{u}\left(\sigma^{\prime}\right)=\mathrm{u}(\sigma)=\sigma_{i}$, by Lemma 5.5; so $\sigma^{\prime}<\sigma_{i}$. Thus $\operatorname{supp}(\delta \sigma) \subseteq \operatorname{supp}\left(d_{\oplus} \sigma\right)$.

Conversely, let $\sigma^{\prime \prime} \in \operatorname{supp}\left(d_{\oplus} \sigma\right)$. Then $\sigma^{\prime \prime} \in S_{i}$, so u $\left(\sigma^{\prime \prime}\right)=\sigma_{i}$, by Lemma 5.5; and $\sigma^{\prime \prime} \leq \sigma_{i}=\mathrm{u}(\sigma)$. Hence $\operatorname{supp}\left(d_{\oplus} \sigma\right) \subseteq \operatorname{supp}(\delta \sigma)$, which proves the lemma.

\section{Corollary 5.7.}

(1) $\left(C^{*}(\Sigma), \delta\right)$ is a cochain complex, and ८ induces an isomorphism of cochain complexes

$$
\iota^{*}:\left(C^{*}(\Sigma), \delta\right) \approx\left(C^{*}(\oplus), d_{\oplus}\right) .
$$

(2) เ induces an isomorphism in cohomology

$$
\iota^{*}: H^{*}\left(C^{*}(\Sigma), \delta\right) \approx H^{*}\left(C^{*}(\oplus), d_{\oplus}\right) .
$$

End of proof of Theorem 5.2. We now return to the proof of Theorem 5.2.

By Lemma 5.4, for $i=1, \ldots, q-1, \Sigma(i-1)$ is a deformation retract of $\Sigma(i)$; and it follows that

$$
H^{*}\left(C^{*}(i), d_{i}\right)=H^{*}(\Sigma(i), \Sigma(i-1))=0
$$

(in all degrees). Now

$$
\begin{aligned}
H^{*}\left(C^{*}(\oplus), d_{\oplus}\right) & =\bigoplus_{i=1}^{q} H^{*}\left(C^{*}(i), d_{i}\right) \\
& =\bigoplus_{i=1}^{q} H^{*}(\Sigma(i), \Sigma(i-1)) \\
& =H^{*}(\Sigma(1), \Sigma(0)) \quad(\text { by the last observation) } \\
& =H^{*}(\Sigma(1)) \quad(\text { since } \Sigma(0)=\emptyset) .
\end{aligned}
$$

Hence

$$
H^{*}\left(C^{*}(\oplus), d_{\oplus}\right)=H^{*}(\Sigma(1))= \begin{cases}\mathrm{Z} & \text { in degree } 0, \\ 0 & \text { in all other degrees. }\end{cases}
$$

Applying Corollary 5.7(2) completes the proof of the theorem. 


\section{Chain Complex of Matroids}

One more result of the present paper clarifies the nature of the chain complex of Coxeter matroids constructed in [6]. Let $W$ be a finite Coxeter group of rank $n$ with the standard generators $r_{1}, \ldots, r_{n}$. Every matroid $\mu$ for $W$ and a parabolic subgroup $P=\left\langle r_{i_{1}}, \ldots, r_{i_{k}}\right\rangle$ was interpreted in [6] as some set of faces of dimension $k=\operatorname{rank}(P)$ of a generalised permutahedron $\Pi$ for $W$ viewed as a reflection group. (The number $k=\operatorname{rank}(P)$ is called the Coxeter rank of $\mu$.) If we denote by $\partial \mu$ the set of all faces of dimension $k-1$ on $\Pi$ adjacent to faces in $\mu$, then it is proven in [6] that $\partial \mu$ is the disjoint union of sets of faces of matroids, denoted $\partial_{Q_{i}} \mu$, for $W$ and the parabolic subgroup $Q_{i_{j}}=\left\langle r_{i_{1}}, \ldots, \widehat{r_{i_{j}}}, \ldots, r_{i_{k}}\right\rangle$ (here we slightly deviate from the notation in [6]) and $\partial_{Q_{i j}} \mu$ has Coxeter rank $k-1$. The paper [6] introduces a free $\mathbb{Z} / 2 \mathbb{Z}$ module $\mathbf{M}$ generated by all matroids for $W$ and all its parabolic subgroups, with the natural grading by their Coxeter rank. It was proven [6, Theorem 3] that the map

$$
\partial: \mu \mapsto \sum_{i_{j} \in\left\{i_{1}, \ldots, i_{k}\right\}} \partial_{Q_{i_{j}}} \mu
$$

is a differential on $\mathbf{M}$. If one compares the interpretation of the chain complex $C(\mathbf{M}, \partial)$ given in [6] with the construction of the coboundary operator $\delta$ on the cochain complex $\left(C^{*}(\Sigma), \delta\right)$ introduced in Section 5, one can immediately see that

$$
\partial_{Q_{i_{j}}} \mu=\mu \vee \mu^{P_{i_{j}}}
$$

and that the set of matroids $\partial_{Q_{i j}} \mu$ is exactly the set of all simplices of dimension $\operatorname{dim} \mu+1$ in $\Omega[u(\mu)]$ which contain $\mu$. This means that the chain complex $C(\mathbf{M}, \partial)$ is obtained from the cochain complex $\left(C^{*}(\Sigma), \delta\right)$ by reversing the indices, $i \mapsto n-1-i$, and taking the coefficients modulo 2. In particular, we have, as a corollary of Theorem 5.1, the following result, answering a question in [6].

Theorem 6.1. The chain complex $C(\mathbf{M}, \partial)$ has the following homology groups:

$$
H_{*}(C(\mathbf{M}, \partial))= \begin{cases}\mathbb{Z} / 2 \mathbb{Z} & \text { in degree } n-1, \\ 0 & \text { in all other degrees }\end{cases}
$$

\section{Acknowledgment}

The authors thank a referee whose comments lead to considerable improvement of proofs and results in the paper.

\section{References}

1. T. V. Alekseyevskaya, A. V. Borovik, I. M. Gelfand and N. White, Matroid homology, in The Gelfand Mathematical Seminar, 1996-1999 (I. M. Gelfand and V. S. Retakh, eds.), Birkhäuser, Boston, 1999, pp. 1-13. 
2. A. Björner, Shellable and Cohen-Macauley partially ordered sets, Trans. Amer. Math. Soc. 360 (1980), $159-183$.

3. A. Björner, Some combinatorial and algebraic properties of Coxeter complexes and Tits buildings, $A d v$. in Math. 52 (1984), 173-212.

4. A. Björner, Homology and shellability, in Matroid Applications, N. White, ed., Cambridge University Press, Cambridge, 1992.

5. A. Björner and M. Wachs, On lexicographically shellable posets, Trans. Amer. Math. Soc. 277 (1983), 323-341.

6. A. V. Borovik, I. M. Gelfand and N. L. White, Boundaries of Coxeter matroids, Adv. in Math. 120 (1996), 258-264.

7. A. V. Borovik, I. M. Gelfand and N. White, Flag matroids, Manchester Centre for Pure Mathematics, Preprint 1997/17, 24 pp.

8. A. V. Borovik, I. M. Gelfand and N. White, On exchange properties of Coxeter matroids and oriented matroids, Discrete Math. 179 (1998), 59-72.

9. A. V. Borovik, I. M. Gelfand and N. White, Combinatorial geometry in characteristic 1, Manchester Centre for Pure Mathematics, Preprint 1999/10, 30 pp.

10. A. V. Borovik, I. M. Gelfand and N. White, Combinatorial flag varieties, J. Combin. Theory Ser. A 91 (2000), 111-136.

11. A. V. Borovik, I. M. Gelfand and N. White, Representations of matroids in semimodular lattices, European J. Combin., to appear.

12. A. V. Borovik, I. M. Gelfand and N. White, Coxeter Matroids, Birkhauser, Boston, in preparation.

13. A. V. Borovik and K. S. Roberts, Coxeter groups and matroids, in Groups of Lie Type and Geometries, W. M. Kantor and L. Di Martino, eds., Cambridge University Press, Cambridge, 1995, pp. 13-34.

14. O. Boruvka, O jistém problému minimálním, Prace Mor. Př́rodově Spol. Brně (Acta Societ. Scient. Natur. Moravicae) 3 (1926), 37-58.

15. K. S. Brown, Buildings, Springer-Verlag, New York, 1989.

16. V. V. Deodhar, Some characterizations of Bruhat ordering on a Coxeter group and determination of the relative Möbius function, Invent. Math. 39 (1977), 187-198.

17. V. V. Deodhar, A splitting criterion for the Bruhat ordering on Coxeter groups, Comm. Algebra 15 (1987), 1889-1894.

18. D. Gale, Optimal assignments in an ordered set: an application of matroid theory, J. Combin. Theory 4 (1968), 1073-1082.

19. I. M. Gelfand and V. V. Serganova, On a general definition of a matroid and a greedoid, Soviet Math. Dokl. 35 (1987), 6-10.

20. I. M. Gelfand and V. V. Serganova, Combinatorial geometries and torus strata on homogeneous compact manifolds, Russian Math. Surveys 42 (1987), 133-168; see also I. M. Gelfand, Collected Papers, vol. III, Springer-Verlag, New York, 1989, pp. 926-958.

21. M. Kontsevich, Formal (non)-commutative symplectic geometry, in The Gelfand Mathematical Seminars, 1990-1992, Birkhäuser, Boston, 1993, pp. 173-187.

22. M. Kontsevich, Feynman diagrams and low-dimensional topology, in First European Congress of Mathematics, Paris, July 6-10, 1992, Vol. 2, Birkhäuser, Boston, 1994, pp. 97-122.

23. D. Kozlov, General lexicographic shellability and orbit arrangements, Ann. Combinatorics 1 (1997), 6790.

24. J. P. S. Kung, Strong maps, in Theory of Matroids, N. White, ed., Cambridge University Press, Cambridge, 1986, Chapter 8.

25. L. Solomon, The Steinberg character of a finite group with $B N$-pair, in Theory of Finite Groups. Proc. Sympos. Harvard 1968, R. Brauer and C. H. Sah, eds., Benjamin, New York, 1969, pp. 213-221.

26. J. Tits, A local approach to buildings, in The Geometric Vein (Coxeter Festschrift), Springer-Verlag, New York, 1981, pp. 317-322.

27. N. White, ed., Theory of Matroids, Cambridge University Press, Cambridge, 1986.

28. N. White, Oral communication.

Received January 16, 2000, and in revised form October 7, 2000, and April 16, 2001.

Online publication December 21, 2001. 